\title{
DIRECT PHOTON PRODUCTION IN POLARIZED PP COLLISIONS
}

\author{
Edmond L. Berger* \\ High Energy Physics Division, Argonne National Lab, Argonne, IL 60439 \\ Jianwei Qjut \\ Institute for Theoretical Physics, SUNY, Stony Brook, New York 11794-3840
}

\begin{abstract}
We argue that inclusive direct photon production at large transverse momentum in proton-proton interactions with longitudinally polarized beam and target is an incisive probe of the polarized gluon distribution in a proton. We provide predictions of cross sections for a range of reasonable choices of the polarized gluon distribution. At current fixed target energies, the cross sections are small but measurable. At collider energies, such as the SSC energy, isolated direct photon production should be considered in order to minimize the nonperturbative contribution due to fragmentation.
\end{abstract}

\section{DISCLAIMER}

This report was prepared as an account of work sponsored by an agency of the United States Government. Neither the United States Government nor any agency thureof, nor any of their employees, makes any warranty, express or implied, or assumes any legal liability or responsibility for the accuracy, completeness, or usefulness of any information, apparatus, product, or process disclosed, or represents that its use would not infringe privately owned rights. Reference herein to any specilic commercial product, process, or service by trade name, trademark, manufacturer, or otherwise does not necessarily constitute or imply its endorsement, recommendation, or favoring by the United States Government or any agency thereof. The views and opinions of authors expressed herein do not necessarily state or reflect those of the United States Government or any agency thereof.

\footnotetext{
-Work supported by the U.S. Department of Energy, Division of High Energy Physics, Contract W-31-109-ENG-38.

'SSC Fellow; Research at SUNY is supported in part by the National Science Foundation under grant No. PHY-89-08495.
} 
ANL-HEP-CP-91-01

$1 T P-S B-90-102$

\title{
DIRECT PHOTON PRODUCTION IN POLARIZED PP COLLISIONS*
}

\author{
Edmond L. Berger \\ High Energy Physics Division, Argonne National Lab, Argonne, IL 60439 \\ Jianwei Qiu \\ Institute for Theoretical Physics, SUNY, Stony Brook, New York 11794-3\$40
}

\begin{abstract}
We argue that inclusive direct photon production at large transverse momentum in proton-proton interactions with lengitudinally polarized beam and target is an incisive probe of the polarized gluon distributien in a proton. We provide predictions of cross sections for a range oi reasonable choices of the polarized gluon distribution. At current fixed target energies, the cross sections are small but measurable. At collider energies, such as the SSC energy, isolated direct photon producticn should be considered in order to minimize the nonperturbative contribution due to fragmentation.
\end{abstract}

\section{INTRODUCTION}

Recent data on the polarization asymmetry in deeply inelastic scattering (DIS) of polarized muons on polarized protons have renewed interest in the relationship between the spin of a proton and the polarization of its constituents (quarks and gluons). ${ }^{1,2}$ This asymmetry measures the spin-dependent structure function $g_{1}^{P}\left(x, Q^{2}\right)$ of a proton. ${ }^{1}$ Here $x$ is the usual Bjorken scaling rariable, and $Q^{2}$ is the square of the four-momentum transfer. The measurement of $g_{1}^{p}\left(x, Q^{2}\right)$ and its first moment, combined with the Bjorken sum rule and the $F / D$ ratio obtained from semileptonic hyperon decays, permits a determination of the first moment of the SU(3)-singlet combination of the spin-dependent quark distributions, $\Delta \Sigma$. The result is that $\Delta \Sigma$ is consistent with zero. ${ }^{1}$ This result has led to a good deal of discussion in the literature. ${ }^{3}$ At the core of the discussion is how to interpret (or to define) the "right" spin-dependent quark distributions, $\Delta g\left(x, Q^{2}\right)$, and whether there is a large gluonic contribution to the first moment of $g_{1}^{P}\left(x, Q^{2}\right)$. Of particular interest to us is the spin-dependent gluon distribution in a polarized proton and its independent experimental neasurament. ${ }^{4}$

Direct access to the spin-dependent gluon distribution should be provided by processes in which the gluons contribute dominantly to the cross sections. In the case of unpolarized proton-proton scattering, direct photon production at large transverse momentum, $p_{T}$, is known to be dominated by the "Compton" subprocess $q g \rightarrow \gamma q$ at fixed target energies. ${ }^{5}$ Here $q$ and $g$ stand for quark and gluon constituents. Da$\mathrm{ta}$, therefore, allow a direct determination of the (spin-averaged) gluon distrabution $G\left(x, Q^{2}\right)$, provided the quark distributions are well-determined in other processes (e.g., in DIS). When both the beam and target protons are polarized, the cross section due to the "Compton" subprocess can be expressed directly in terms of the polarized gluon distribution $\Delta G\left(x, Q^{2}\right){ }^{6}$ Based on the DIS data and a range of reasonable choices of spin-dependent gluon distributions, we shall argue that the inclusive direct photon production in polarized $p p$ collisions is also dominated by the "Compton" subprocess. As a result, such experiments will provide direct access to the spin-dependent givon distribution in a polarized proton.

\footnotetext{
"Invited talk presented at the Polarized Collider Workshop, Novermber 15-17, 1990, Pennsylvania State University, University Park, PA.

'SSC Fellow
} 
In Sec. II we explain why the "Compton" subprocess dominates the cross section for unpolarized hadronic prompt photon production. We then argue that the same conclusion can be reached for the polarized case. In Sec. III we provide specific predictions for the cross sections and polarization asymmetries expected for direct photon production in polarized $p p$ collisions at fixed target energies. We discuss our expectations for polarized direct photon production at collider energies in Sec. TV. As shown explicitly in Sec. IV, at leading order, our predictions are not very sensitive to what interpretation or definition one adopts for the spin-dependent quark distributions. Instead, our prediction is directly related to the experimentally measured structure function $g_{1}^{p}\left(x, Q^{2}\right)$ as a whole. Conclusions are also found in Sec. IV.

\section{PROMPT PHOTONS AT LARGE $p_{T}$}

Measurement of inclusive direct photon production at large $p_{T}$ in $p p$ collisions is known to be a clean probe of the (spin-averaged) gluon distribution in a proton at fixed target energies. However, at collider energies, isolated (instead of inclusive) direct. photon production must be considered in order to minimize the large contril'ation from nonperturbative photon fragmentation. ${ }^{7}$ Many estimates are available of cross sections for direct photon production in unpolarized pp collisions. ${ }^{5}$ Data are generally in accord with the calculations. To understand how good direct photon production in polarized $p p$ collisions may be in determining the spin-dependent gluon distribution, it is important to examine why the process is good for determining the gluon distribution in the case of unpolarized $p p$ collisions.

At large $p_{T}$, a photon can be produced through a pointlike, perturbatively calculable hard scattering term, as well as through a nonperturbative fragmentation process (generalization of the bremsstrahlung process). Because of the nonperturbative nature of the fragmentation process, perturbative QCD alone cannot predict the actual size of the inclusive prompt photon cross section. In principle, whether in the case of unpolarized or polarized interactions, we can expect direct photon production to give good information on parton (gluon or quark) distributions only if the nonperturbative fragmentation process can be controlled.

With approximate leading logarithmic photon fragmentation functions, we estimate that the fragmentation contribution in the unpolarized case is only about a $10 \%$ effect at current fixed target energies. In contrast, it amounts to a $50 \%$ (or even larger) effect at collider energies. ${ }^{7}$ Because of the poorly known photor fragmentation function, we do not expect that inclusive direct photon production at-high energy can yield clean information on gluon distributions. However, at fixed target energies, inclusive prompt photon is a relatively good probe of short-distance physics.

At leading order, short-distance prompt photon production is governed by the tree "Compton" $q g \rightarrow \gamma q$ and annihilation $q \tilde{q} \rightarrow \gamma g$ subprocesses. The "Compton" (or annihilation) contribution to the cross section can be written in general as

$$
\begin{aligned}
& \sigma_{\text {Com }}=(q \otimes G) \otimes \hat{\sigma}_{\text {Com }} \text { "Compton" } \\
& \sigma_{\text {anni }}=(q \otimes \bar{q}) \otimes \hat{\sigma}_{\text {anni }} \text { Annihilation, }
\end{aligned}
$$

where $\hat{\sigma}$ is a parton level cross section - the short-distance hald scattering term, $q$ $(\bar{q})$ and $g$ are quark (antiquark) and gluon distributions, and $\otimes$ means a convolution between the distributions and the hard scattering term. The expression in parentheses () can be thought of as the parton flux. The statement that inclusive direct photon production is a good piobe of the gluon distribution for the unpolarized case is based on the fact that the net "Compton" contribution in $p p$ interactions is numerically much larger than the annibilation contribution. At the parton level, the "Compton" contri- 


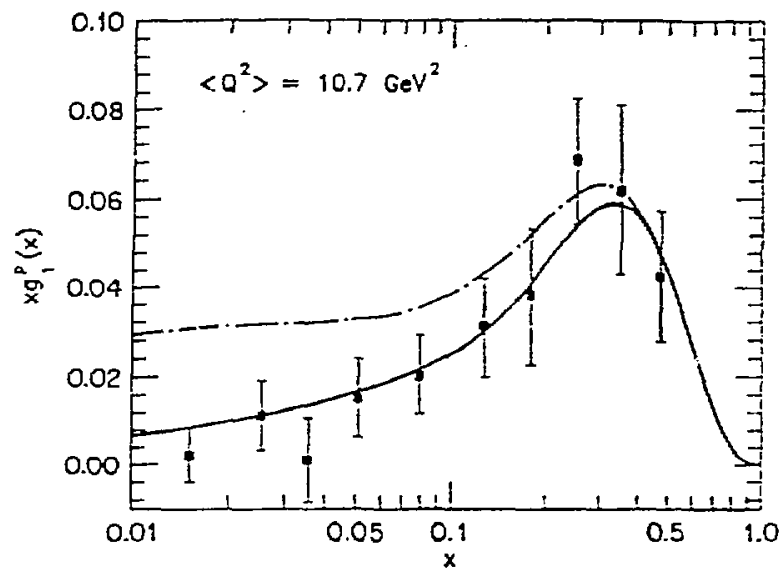

Figure 1: The dot-dashed line is the contribution to $x g_{1}^{p}\left(x, Q^{2}\right)$ from the polarized valence quark distributions at $Q^{2}=10.7 \mathrm{GeV}^{2}$. The solid line is our parametrization of $x g_{1}^{P}\left(x, Q^{2}\right)$ including effective sea distributions (Ref. 4$)$. The data are from ENIC (Ref. $:$ ).

bution is actually smaller than the annihilation contribution, $\hat{\sigma}_{\text {Com }}<\hat{\sigma}_{\text {anni }}$. The reason the net "Comptcn" contribution is larger than the annibilation contribution is that in a proton (spin-averaged) the gluon density is much larger than the antiquark density. Equivalently, the difference in the parton fiux controls the answer. For example, if $x_{T}=2 p_{T} / \sqrt{s}$ and $x_{a} \sim x_{b} \sim x_{T} \sim 0.1, \hat{\sigma}_{\text {Com }} \sim(1 / 2) \hat{\sigma}_{\text {anni; }}$ but $G(x) \sim 20 \bar{q}(x)$ for $x \sim 0.1$. Here $x_{a}$ and $x_{b}$ are momentum fractions carried by initial partons participating in the hard scattering. Because the observed cross section is dominated by the region where $x_{a} \sim x_{b} \sim x_{T}$, the above simple estimate suggests that $\sigma_{\mathrm{Cam}} \sim 10 \sigma_{\text {anni, }}$, about the same as is obtained in a more precise numerical calculation.

In the case of polarized interactions, the hard part for the annihilation subprocess is the same as that for the unpolarized case up to a sign difference. But, the hard part for the "Compton" subprocess is even smaller than that in the unpolarized case. As a result, $\Delta \hat{\sigma}_{\text {Com }} \sim(1 / 4)\left|\Delta \hat{\sigma}_{\text {anni }}\right|$ for $x_{a} \sim x_{b} \sim x_{T} \sim 0.1$. Therefore, inclusive direct photon production in polarized $p p$ collisions can yield useful information on $\Delta G$ iff $\Delta G$ is much bigger than $\Delta \bar{q}$, which is not a priori obvious. The spin-averáged gluon distribution is much larger than the antiquark distribution because the evolution lernel for gluons has a $1 / x$ pole. In contrast, the evolution kernel for the spin-dependent gluon distribution does not have the $1 / \pi$ pole. Consequently, $\Delta G$ is much smaller than $G$ when $x$ is small. The question is now whether $\Delta \vec{q}$ is even smaller.

To estimate the size of $\Delta \bar{q}_{1}$ we assume that the Carlitz and Kaur ${ }^{8}$ model is approximately valid for the spin-dependent valence quark distributions. We justify this assumption by noting that the valence quark part of the structure function $\left.g_{1}^{p}\left(x, Q^{2}\right)\right|_{\text {val }}$, the dot-dashed curve in Fig. 2, fits the data in the large $x$ region. Then, we use the difierence between the data and $\left.g_{1}^{p}\left(x, Q^{2}\right)\right|_{\text {val }}$ in the small $x$ region to parametrize the effective spin-dependent sea and anti-quark distributions. From Fig. 1, for example, 
we can estimate $\Delta \bar{q}(x)$ for $x \sim 0.1$ as follows:

$$
\left.x g_{1}^{P}\left(x, Q^{2}\right)\right|_{\mathrm{data}}-\left.x g_{1}^{p}\left(x, Q^{2}\right)\right|_{\mathrm{val}} \approx 2\left[2\left(\frac{1}{3}\right)^{2}+\left(\frac{2}{3}\right)^{2}\right] x \Delta \bar{q}\left(x, Q^{2}\right)
$$

where $\left.x g_{1}^{p}\left(x, Q^{2}\right)\right|_{\text {data }}$ means the solid curve, and an $\mathrm{SU}(3)$ symmetric sea is assumed. Here the scale $Q^{2}$ is about $10.7 \mathrm{GeV}^{2}$. For $x \sim 0.1,\left.x g_{1}^{p}\left(x, Q^{2}\right)\right|_{\text {data }}-\left.x g_{1}^{p}\left(x, Q^{2}\right)\right|_{\text {Val }} \approx$ 0.01 , and consequently, $\Delta \bar{g}\left(x, Q^{2}\right) \sim-0.075$ when $x \sim 0.1$.

Because $\triangle G\left(x, Q^{2}\right)$ should be much smaller than $G\left(x, Q^{2}\right)$ in the small $x$ region, a conservative assumption is that $\Delta G\left(x, Q^{2}\right) \approx x G\left(x, Q^{2}\right)$. Taking $x G\left(x, Q^{2}\right) \sim 3(1-x)^{5}$, we have $\Delta G\left(x, Q^{2}\right) \sim 1.8$ for $x \sim 0.1$. Comparing with $\left|\Delta \tilde{q}\left(x, Q^{2}\right)\right| \sim 0.075$, we observe that $\Delta G\left(x, Q^{2}\right)$ is much larger than $\Delta \bar{q}\left(x, Q^{2}\right)$ in the region where $x \sim 0.1$. We conclude that inclusive direct photon production in polarized pp collisions is a good probe of the spin-depindent gluon distribution, $\Delta G\left(x, Q^{2}\right)$, of a polarized proton.

\section{NUMERICAL RESULTS}

To obtain quantitative predictions for the cross sections for inclusive direct phoion production in polarized $p p$ collisions, we need parametrizations of spin-dependent quark and gluon distributions. The general procedure to obtain the spin-dependent parton distributions is first to parametrize the distributions at an arbitrary reference value $Q^{2}=Q_{0}^{2}$, and then to use the spin-dependent Altarelli-Parisi evolution equations ${ }^{9}$ to obtain the distributions at any other scale $Q^{2}$. To parametrize the spin-dependent input parton distribui, sns, we adopt the Carlitz and haur model for spin-dependent valence quark distributions. With the assumption of an $S U(3)$ symmetric sea, we parametrize the spin-dependent sea quark and antiquark distributions by fitting the difference between the data and the prediction from the Carlitz and Kaur model. For the spin-dependent gluon distribution $\Delta G\left(x, Q^{2}\right)=G^{(+)}\left(x, Q^{2}\right)-G^{(-)}\left(x, Q^{2}\right)$, the difference between the numbers of gluons with positive and negative helicity, we select a simple one-parameter function ${ }^{4}$

$$
\Delta G\left(x, Q_{0}^{2}\right)= \begin{cases}G\left(x, Q_{0}^{2}\right), & x_{c} \leq x \leq 1 \\ \frac{x}{x_{c}} G\left(x, Q_{0}^{2}\right), & 0 \leq x \leq x_{c}\end{cases}
$$

The parameter $x_{c}$ with $0<x_{c} \leq 1$ determines the net helicity carried by gluons. When the value of $x_{c}$ is adjusted, this simple parametrization covers a spectrum of reasonable choices of the polarized gluon distribution. The behavior of our polarized parton distributions is displayed numerically in Ref. 4.

In leading-order QCD perturbation theory, the cross section for prompt photon production in polarized $p p$ reactions, $\vec{p}_{A}+\vec{p}_{B} \rightarrow \gamma+X$, is given by

$$
\begin{gathered}
E_{\gamma} \frac{d \Delta \sigma_{A B}}{d^{3} p_{\gamma}}\left(s, x_{F}, p_{T}\right)=\sum_{a, b} \int d x_{a} d x_{b} \Delta P_{A}^{a}\left(x_{a}, Q^{2}\right) \Delta P_{B}^{b}\left(x_{b}, Q^{2}\right) \\
\times E_{\gamma} \frac{d \Delta \hat{\sigma}_{a b}}{d^{3} p_{\gamma}}\left(\hat{s}, x_{F}, p_{T}\right),
\end{gathered}
$$

where $a$ and $b$ run over all possible quark flavors and the gluon; $\Delta \sigma_{p p} \equiv \frac{1}{2}\left[\sigma_{p p}(++)-\right.$ $\left.\sigma_{p p}(+-)\right] ;$ and $\sigma_{p p}(++)$ denotes the cross section when the two incident protons have their spins oriented along the directions of motion of the protons in the overall $p p$ center-of-mass system. Similarly, for the unpolarized case,

$$
E_{\gamma} \frac{d \sigma_{A B}}{d^{3} p_{\gamma}}\left(s, x_{F}, p_{T}\right)=\sum_{a, b} \int d x_{a} d x_{b} P_{A}^{a}\left(x_{a}, Q^{2}\right) P_{B}^{\delta}\left(x_{b}, Q^{2}\right) E_{\gamma} \frac{d \bar{\sigma}_{a b}}{d^{3} p_{\gamma}}\left(\hat{s}, x_{F}, p_{T}\right) .
$$




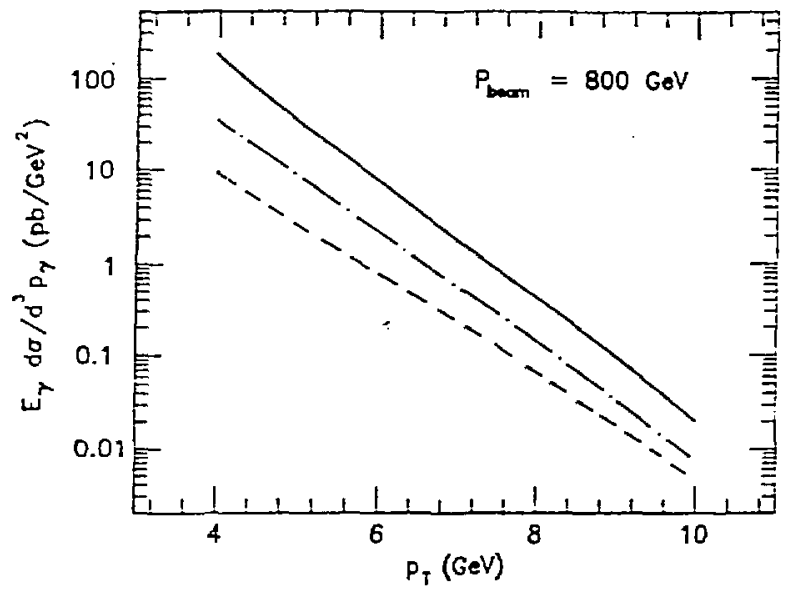

Figure 2: Predicted cross sections for prompt photons as a function of $p_{T}$ for $x_{F}=0$ in $p p$ interactions at pbeam $=800 \mathrm{GeV} / c$. The solid line shows the rate in unpolarized pp interaction, whereas the dashed and dot-dashed curves show a range of rates in the polarized case.

In Eq. (4), $\Delta P_{A}^{a}\left(x_{a}, Q^{2}\right)$ and $\Delta P_{B}^{b}\left(x_{a}, Q^{2}\right)$ are spin-dependent parton distributions; and in Eq. (5), $P_{A}^{c}\left(x_{a}, Q^{2}\right)$ and $P_{B}^{b}\left(x_{a}, Q^{2}\right)$ are spin-averaged parton distributions. The short-distance hard scattering terms $\Delta \bar{\sigma}$ and $\hat{\sigma}$ include the parton level "Compton" and annihilation subprocesses. Their analytical expressions can be found in Ref. 4 .

Having the parton distributions and expressions for the cross sections, we can calculate rates for direct photon production at different energies. ${ }^{4}$ In Fig. 2 we plot the production rates as a function of the photon's transverse momentum $p_{T}$ for incoming beam energy eçual to $800 \mathrm{GeV}^{2}$. The solid line represents the rate in unpolarized $p p$ collisions. The dashed line represents the rate in polarized $p p$ interactions with parameter $x_{c}=1.0$ for the polarized input gluon distribution, and dot-dashed line for $x_{c}=0.2$. These two lines in the polarized case represent roughly a reasonable range expected for the polarized cross section at fixed target energies. They correspond to values of the integral (integral of $\Delta G$ over all $x$ ) of 0.5 and 2.5 at $Q \sim 2 \mathrm{GeV}$. The predicted energy dependence of the cross sections is shown in Fig. 3. Notice in Fig. 3 that when energy increases, the unpolarized cross section increases faster than the polarized one. At higher energies, smaller values of $x$ contribute, and at small $x$, the unpolarized gluon distribution is much larger than its polarized counterpart. From Fig. 2, we notice that the cross section is small for direct photon production with transverse momentum $p_{T}>4 \mathrm{GeV}$, but it is still measurable.

\section{DISCUSSION AND CONCLUSIONS}

We have argued that at fixed target energies, inclusive direct photon production in polarized $p p$ collisions is a good probe of the spin-dependent gluon distribution in a proton. At collider energies (e.g., SSC energy), the inclusive process is no longer ideal for measuring the gluon distribution because of the large nonperturbative contribution due to fragmentation processes. However, our experience with the treatment of the 


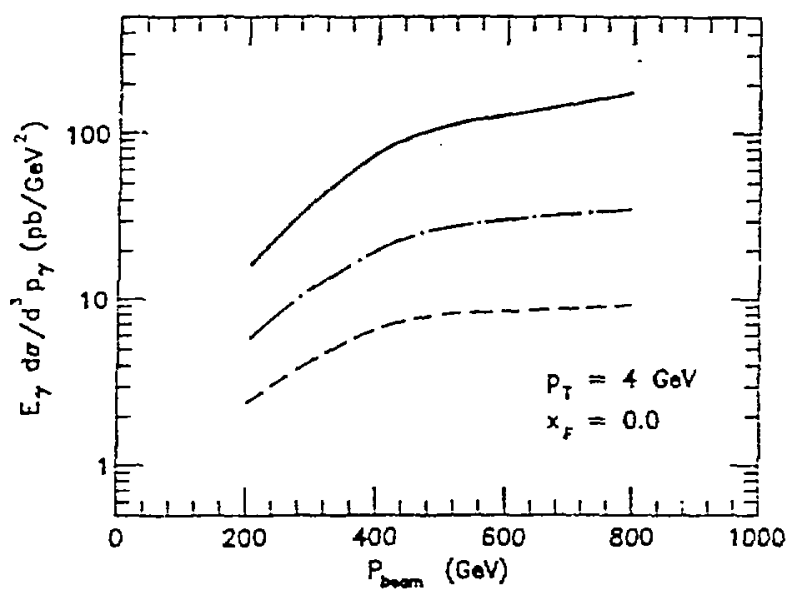

Figure 3: Predicted cross sections for prompt photons as a function of beam energy $p_{\text {beam }}$ for $x_{F}=0$ and $p_{T}=4 \mathrm{GeV}$. The solid, dashed and dot-dashed lines are defined as in Fig. 2.

unpolarized case ${ }^{i}$ at collider energies leads us to believe that isolated direct photon production in polarized $p p$ interactions should be an appropriate process from which to extract the spin-dependent gluon distribution, as long as the isolation cut is imposed properly in both experimental measurements and theoretical calculations.

Our numerical predictions for the rate of direct photon production at large $p_{T}$ depend on the polarized parion distributions. Experiments measure only a combination of the distributions, for example, the structure function $g_{1}^{P}\left(x, Q^{2}\right)$. One question of interest is how sensitively our predictions depend on the different parametrizations (or separations) of the valence and sea quark distributions. Our conclusion is that at leading order, the cross section is actually insensitive to the different choices of parametrizations of parton distributions, as long as these distributions fit the DIS data. This result is due to dominance of the "Compton" subprocess. To make our point more transparent, we rewrite the cross sections given in Eqs. (4) and (5) approximately as

$$
\begin{gathered}
E_{\gamma} \frac{d \Delta \sigma_{A B}}{d^{3} p_{\gamma}}\left(s, x_{F}, p_{T}\right)=\int d x_{a} d x_{b}\left[2 g_{1}^{p}\left(x_{a}, Q^{2}\right) \Delta G\left(x_{b}, Q^{2}\right) E_{\gamma} \frac{d \Delta \tilde{\sigma}_{q g}}{d^{3} p_{\gamma}}\left(\hat{s}_{;}, x_{F}, p_{T}\right)\right. \\
\left.+\left(x_{a} \leftrightarrow x_{b}\right)\right]+ \text { “Annihilation term" }
\end{gathered}
$$

and

$$
\begin{aligned}
E_{\gamma} \frac{d \sigma_{A B}}{d^{3} p_{\gamma}}\left(s, x_{F}, p_{T}\right)=\int & d x_{a} d x_{b}\left[\frac{F_{2}\left(x_{a}, Q^{2}\right)}{x_{a}} G\left(x_{b}, Q^{2}\right) E_{\gamma} \frac{d \bar{\sigma}_{q g}}{d^{3} p_{\gamma}}\left(\hat{s}_{,} x_{F}, p_{T}\right)\right. \\
& \left.+\left(x_{a} \leftrightarrow x_{b}\right)\right]+ \text { "Annihilation term" }
\end{aligned}
$$

where $g_{1}^{P}\left(x, Q^{2}\right)$ and $F_{2}\left(x, Q^{2}\right)$ are the two proton structure functions measured in polarized and unpolarized DIS. Neglecting the annihilation terms, one sees immediately that the cross sections for direct photon production are proportional to measured structure 
functions and are therefore not sensitive to the detailed parametrizations of the quark distributions of different flavors. More important is that Eqs. (6) and (7) show that large $p_{T}$ direct photon production in $p p$ interactions measures the gluon distribution directly, provided the proton structure functions are well measured in DIS.

The $x$ range explored by fixed target experiments is limited. Based on fixed target data, it will be difficult to derive an accurate measurement of the first moment of the polarized gluon distribution, which we would like to know. Nevertheless the sign of the polarized gluon distribution as well as its magnitude in the large and intermediate $x$ regions should be accessible experimentally: This information would be particularly interesting.

We conclude that inclusive direct photon production at large $p_{T}$ in polarized $p p$ collisions at fixed target energies is dominated by the gluon-induced "Compton" subprocess. Correspondingly, fixed target experiments with longitudinally polarized proton beams and targets would measure the sign and magnitude of the polarized gluon distribution in a proton in interesting regions of $x$. At fixed target energies, the cross section is small, but it should be measurable. The production rate will be greater at higher energies (such as the SSC energy), but at ccllider energies an isolation cur on the photon may be required to minimize the nonperturbative contribution due to fragmentation processes.

\section{Acknowledgements}

We have benefitted from discussions with J.C Collins, G. Sterman, and W.I. Tung. The research was supported in part by the U.S. Department of Energy, Division of High Energy Physics, Contract W-31-109-ENG-38; and by the National Science Foundation under grant No. PHY-89-08495.

\section{REFERENCES}

1. European Muon Collaboration, J. Asham et al, Phys. Lett. 206B, 364 (1988).

2. M.J. Alguard et al, Phys. Rev. Lett. 37, 1258 (1976); 41, 70 (1978); G. Baum et al, ibid. 51,1153 (1983).

3. For example, see articles in these proceedings by A. Efremov, by J. Manohar, and by G. Bodwin, respectively.

4. E.L. Berger and J. Qiu, Phys. Rev. D40, 778 (1989); D40, 3128 (1989).

5. E.L. Berger, E. Braaten, and R.D. Field, Nucl. Phys. B239; 52 (1984), and references therein; J.F. Owens, Rev. Mod. Phys. $\underline{59}, 465$ (1987).

6. N.S. Craigie, K. Hidaka, M. Jacob, and F.M. Renard, Phys. Rep. 99C, 69 (1983); C. Bourrely, F.M. Renard, J. Soffer and P. Taxil, Phys. Rep. 177, 319 (1989).

7. E.L. Berger-and J. Qiu, Phys. Lett. 248B, 371 (1990); Argonne preprint ANLHEP-PR-90-104.

8. R. Carlitz and J. Kaur, Phys. Rev. Lett. 38,673 (1977).

9. G. Altarelli and G. Parisi, Nucl. Phys. B126, 298 (1977). 